\title{
PERSPECTIVE AND FORM RATIO AS DETERMINANTS OF RELATIVE SLANT JUDGMENTS'
}

\author{
MYRON L. BRAUNSTEIN 2 AND JOHN W. PAYNE \\ University of California, Irvine
}

\begin{abstract}
Judgments of relative slant were elicited by a paired-comparison method from $24 \mathrm{Ss}$ in each of three experiments. The stimuli were computergenerated slides representing regular dot patterns (Exp. I), regular line patterns (Exp. II), or random dot patterns (Exp. III) rotated about a horizontal axis. The ratio of horizontal to vertical separations (form ratio) and perspective were independently varied in Exp. I and II. Perspective clearly dominated slant judgments when in conflict with form ratio as an indicator of degree of slant. Perspective alone was varied in Exp. III and was found to be less effective in determining slant judgments for random dot patterns. The equivalence of perspective and optical theta as explanations of slant perception is discussed.
\end{abstract}

The retinal image of a slanted surface provides at least two potential sources of veridical slant information. The first is the proximal stimulus form, relative to an assumed original form. For a surface rotated about a horizontal axis, form is defined as the ratio of a vertical distance between identifiable features of the surface to a horizontal distance between identifiable features. The vertical distance is measured from the axis of rotation along a line perpendicular to that axis to a horizontal contour line or other identifiable surface feature. The horizontal distance is measured at that vertical distance along a line parallel to the axis of rotation. Then

$$
\cos \theta=\frac{v / h}{V / H},
$$

where $\theta$ is the slant angle, $V$ and $H$ are the vertical and horizontal distances in the original form, and $v$ and $h$ are the corresponding distances in a projection of the slanted surface (see Fig. 1). Flock (1962) and Ericksson (1967) have pointed out that the form ratio depends on viewing distance as well as slant. This is true for their example in which the horizontal distance is taken along the axis of rotation rather than at the opposite terminus of the vertical distance.

\footnotetext{
1 This research was supported by National Science Foundation Grant GB 5545.

${ }^{2}$ Requests for reprints should be sent to Myron L. Braunstein, School of Social Sciences, University of California, Irvine, California 92664.
}

The form ratio, however, is independent of viewing distance when its components are computed in the manner specified.

The second source of slant information may be expressed in several ways. Flock's (1962) optical theta is one such expression:

$$
\cos \theta=\sqrt{\frac{4 K^{2}-\left(1+K^{\prime}\right)^{2}}{4\left(K^{2}-K^{\prime}\right)}},
$$

where $K=\cot \alpha / \cot \gamma, K^{\prime}=\cot \alpha / \cot \phi$, and $\alpha, \gamma$, and $\phi$ are visual angles subtended by three horizontal distances in the projected image, which are equal in the original surface. These horizontal distances must be separated vertically by equal visual angles in the projected image (see Fig. 1). Another expression, based on Freeman's (1966a) presentations, involves the slope of converging lines:

$$
\cot \theta=\tan \pi \tan \gamma,
$$

where $\tan \pi$ is the slope of a projected vertical line and $\gamma$ is the visual angle subtended by the projected distance between this line and the fixation point, along the axis of rotation. A third expression relates the perspective ratio (Braunstein, 1968) to the vertical extent of the projected image :

$$
\tan \theta=\cot \beta \frac{P-1}{P+1},
$$

where $P$ is the ratio of the projections of two horizontal distances that are equal on the original surface and $\beta$ is the visual angle sub- 
tended by half the vertical separation of these distances (see Fig. 1). All three of these expressions are mathematically equivalent descriptions of the pattern of lines radiating from a vanishing point in the projected image and are referred to as perspective.

Form ratio and perspective have important distinguishing characteristics as sources of slant information. The first requires that knowledge of the original form be used in perceptual processing. Veridical slant judgments could then be made solely on the basis of relative dimensions within the retinal image. Such judgments would be possible for photographs, telescopic views, and other artificial projections, as well as for direct vision. The use of perspective as a source of slant information requires the presence on the original surface of parallel vertical lines or of other features that are, on the average, equally spaced in the horizontal dimension. Relative height-width ratios are not involved. This source of information involves relationships among three visual angles in the projected surface and always reduces to relative dimensions in the retinal image with respect to a visual angle. Veridical slant information is provided by this source in direct vision, but not in photographs or other artificial projections unless the distance at which the projection is viewed is equal to the distance between the projection (focal) point and the projection plane used in generating the projection.

The dependence of one of these sources of information on relative dimensions in the original figure and of the other source on a visual angle subtended by a part of the projected image suggests a method of experi- mentally investigating the relative influence of form ratio and perspective on slant judgments. First, a textured surface is rotated about a horizontal axis. When the rotated image is projected onto a frontal plane, the form ratio is equal to the cosine of the angle of rotation. The projected image is then magnified or demagnified until the desired level of perspective is obtained. This is equivalent to changing the projective distance used in generating the display. This process does not affect the form ratio. Any desired level of form ratio, displayed by rotating and projecting a textured surface, can be combined with any desired level of perspective by appropriate magnification or demagnification of the projected image. Slant indications from these two sources, therefore, can be varied independently. The present study tested the hypothesis that perspective rather than the form ratio is the principal source of information used in judgments of relative slant when information from both of these sources is potentially available. The first two experiments tested this hypothesis in regular dot and line patterns. The third experiment examined the effect of perspective on judgments of relative slant in random dot patterns that did not provide form ratio information.

\section{MeTHOD}

\section{Experiment I}

Subjects.-The $S$ s were 24 students in an introductory psychology class who participated as part of a course requirement.

Stimuli.-The stimuli were 120 computer-generated, 35-mm. slides representing each of the possible pairings of 16 displays. The displays consisted of evenly spaced rows and columns of dots, shown
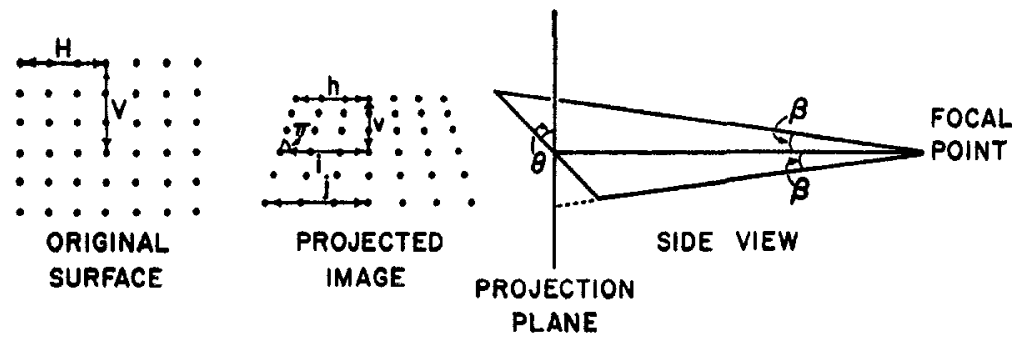

FIG. 1. Geometrical relationships in the projection of a slanted surface. (Segments $h, i$, and $j$ subtend visual angles of $\alpha, \gamma$, and $\phi$, respectively. Segment $i$ is on the axis of rotation. The perspective ratio, $P$, is defined as $j / h$, where these segments represent the vertical limits of $S$ 's field of view.) 


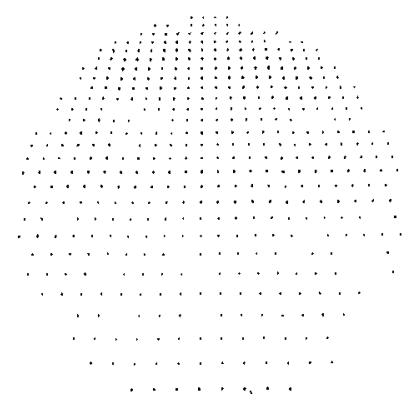

$\theta=25^{\circ}, P=2$

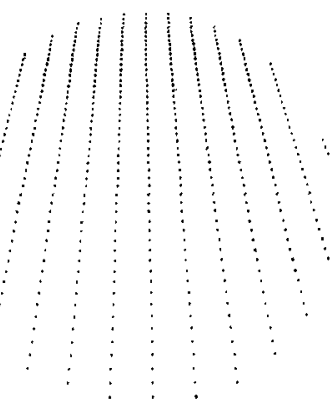

$\theta=75^{\circ}, P=2$

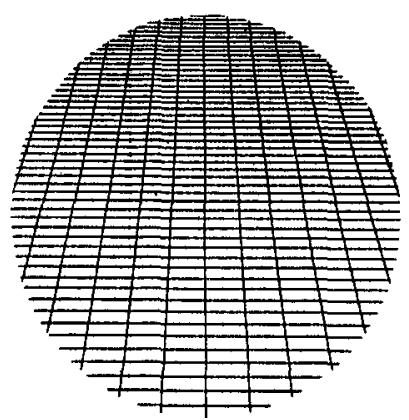

$\theta=75^{\circ}, P=2$

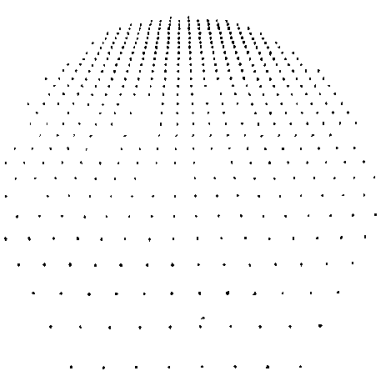

$\theta=25^{\circ}, P=4$

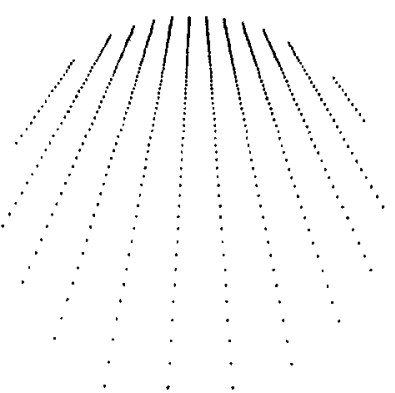

$\theta=75^{\circ}, P=4$

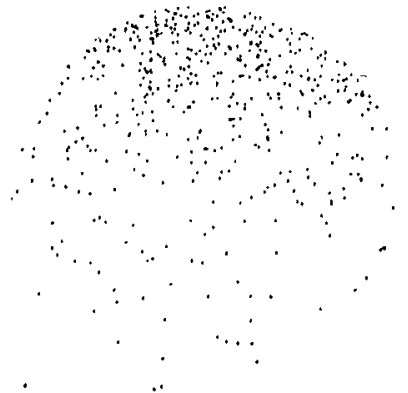

$P=4$

FIG. 2. Examples of stimuli in Exp. I (left and center), Exp. II (upper right), and Exp. III (lower right). ( $\theta$ is the $\cos ^{-1}$ of the form ratio; $P$ is the perspective ratio.)

rotated about a horizontal axis perpendicular to the line of sight. Each display contained $500 \pm 16$ white dots on a black background. Fifteen of the displays presented form ratios 3 equal to the cosines of $25^{\circ}, 50^{\circ}$, and $75^{\circ}$, each with perspective ratios 4 of $1.0,1.5,2.0,3.0$, and 4.0 . One contained the original, even texture. Examples of the stimuli are shown in Fig. 2.

${ }^{3}$ The form ratio can be measured by counting an arbitrary number of dots (Exp. I) or lines (Exp. II) from the center of the projected image along a line perpendicular to the axis of rotation and then counting the same number of elements along a horizontal line. The ratio of the vertical to the horizontal excursion is defined as the proximal stimulus form. It is also the form ratio under the assumption of equal spacing of the elements in the unslanted surface.

${ }^{4}$ Specifically, the perspective ratio is the ratio of the maximum to the minimum separation between projections of columns of dots (Exp. I) or between vertical lines (Exp. II) or the ratio of the maximum to the minimum mean separation between dots in the horizontal dimension (Exp. III).
The following computational procedure was used to determine the coordinates of the displayed dots: First, a form ratio was determined by selecting an angle $\theta$ equal to $0^{\circ}, 25^{\circ}, 50^{\circ}$, or $75^{\circ}$. Then a projection point was computed that would result in the desired perspective ratio, $P$, for the form ratio corresponding to $\theta$. If the radius of the circular viewing area is set equal to one unit, the projection point $(0,0, E)$ is determined by $E=\tan \theta(P+1) /(P$ -1). A set of points was then computed on the $x-y$ plane through the origin, with the points equally spaced along both axes. The points were rotated about the $x$-axis to the angle $\theta$ and projected onto the $x-y$ plane through the origin, using the computed projection point.

Apparatus.-A 35-mm. projector (Kodak Carousel 800) was used to display the slides on a $1.2-\mathrm{m}$. square translucent screen (Polacoat) located between the projector and $S^{\prime}$ s eyes, $92.7 \mathrm{~cm}$. from $S$ 's eyes. At this distance the slants indicated by the five perspective ratios were $0^{\circ}, 36,^{\circ}, 51^{\circ}, 61^{\circ}$, and $65^{\circ}$. The $S$ viewed the screen binocularly through an arrangement of apertures that restricted the field of view of each eye to a separate circular area inscribed within the screen. The images presented to the two eyes did not overlap. A 
distance unit in the stimulus display was $25.4 \mathrm{~cm}$. when the slide was projected onto the screen. Background and dot luminances were approximately .03 and $.3 \mathrm{ftl}$., respectively. The $S^{\prime}$ 's response device was a double-throw momentary rocker switch attached to an automatic recording system. A demonstration device, consisting of a $22.8 \times 14.2 \mathrm{~cm}$. rectangle containing evenly spaced rows and columns of translucent white dots on an opaque black background that could be turned about its horizontal axis, was located to the right of the viewing tube. A 12-v. high-intensity lamp was located directly behind the rectangle.

Procedure.-Each $S$ was given a vision test before entering the experimental room, and all $S_{\mathrm{s}}$ qualified on a criterion of $20 / 30$ or better using a Snellen eye chart; corrected lenses were permitted if they were normally worn. The $S$ s were told they would see a series of displays of white dots on a flat surface, which would be either straight up and down or slanted away from them at the top to varying degrees. The demonstration plane, which was illuminated from the rear, was slanted to angles of approximately $30^{\circ}$ and $60^{\circ}$ to illustrate the instructions. The $S$ s were told that they would see two of the surfaces at a time, one with the right eye and one with the left eye, when looking into the viewing apertures. They were asked to indicate which member of each pair appeared more slanted by pressing the corresponding side of the response switch. A choice was required on each trial.

The slides were presented in blocks of 65 slides and 60 slides, with a 1-2-min. pause between blocks. After 5 practice slides, the 120 stimulus slides were presented to each $S$ in a different random sequence. At the end of the session, 15 of the $S$ s were asked to tilt the demonstration plane to "the greatest degree of slant which [they] saw in any of the displays."

\section{Experiment II}

In the displays of regular dot patterns in Exp. I, variations in form ratio and perspective may affect the perceptual grouping of the dots into horizontal and vertical lines. The effects of proximity grouping were controlled in Exp. II through the substitution of lines for the rows and columns of dots. The lines were arranged so that the positions of their intersections were the same as the positions of the dots in Exp. I.

Subjects.-The $S$ s were 24 students in an introductory psychology class who participated as part of a course requirement. None of these had served in Exp. I.

Stimuli.-The stimuli were similar to those used in Exp. I except that lines were shown in the same positions as the rows and columns of dots in the first experiment (see Fig. 2).

Apparatus and procedure.-The apparatus and procedure were similar to that of Exp. I. Background and line luminances were approximately
.05 and $10 \mathrm{ftl}$, respectively. The $22.8 \times 14.2 \mathrm{~cm}$. rectangle used in Exp. I was replaced by a similarsized rectangle displaying a grid pattern. The instructions were the same except for the substitution of the word "lines" for "dots."

\section{Experiment III}

Experiment III was a further attempt to study the effects of type of texture and perspective on perceived slant, in part to determine whether perspective is a sufficient condition for indicating relative depth. Only perspective was varied since there is no way to display a form ratio in random textures which lack identifiable features.

Subjects.-The $S$ s were 24 students in an introductory psychology class who participated as part of a course requirement. None had served in Exp. I or II.

Stimuli.-The stimuli were 40 computer-generated, $35-\mathrm{mm}$. slides. Each slide represented 1 of the 10 possible pairs of five displays of random dot patterns with perspective values of $1.0,1.5,2.0,3.0$, and 4.0 .

In the computation of these displays, 500 points were randomly distributed on a plane rotated about a horizontal axis. A combination of slant and projection point was selected yielding the desired perspective. (Any such combination would produce the same display from the same random number sequence.) Each display pair was shown four times with different random textures. Five additional practice slides were shown at the beginning of the session. Three $S$ s were shown the 40 slides in each of eight different random orders.

Apparatus and procedure.-The apparatus and procedure were similar to those of Exp. I except for an addition to the instructions regarding the random dot pattern. Five practice slides preceded the presentation of the 40 stimuli.

\section{RESULTS}

Experiment I.-The frequency with which each of the 16 displays was selected as having the greater degree of slant was tabulated for each $S$. The relationship of the mean proportions based on these frequencies to perspective and form ratio is shown in Fig. 3. (The proportion for the frontal view was .08.) The mean of the judgments of greatest displayed slant was $60^{\circ}$.

Experiment $I I$.-The relationship of the mean proportion of trials on which displays were selected as having the greater slant to perspective and form ratio is shown in Fig. 4. (The proportion for the frontal view was .09.) The mean judgment of greatest displayed slant $(N=23)$ was $58^{\circ}$. 
Combined Analyses of Experiments I and $I I$.- In a separate analysis, the 120 pairs of displays in each of the first two experiments were assigned to one of four groups. In Group A, perspective indicated greater slant for one member of the pair, while the form ratio indicated greater slant for the other member. In Group B, greater slant was indicated for the same member of the pair by both sources of information. The form ratio was equal for both members of Group $C$ pairs, while perspective was equal for both members of Group D pairs. The mean proportions of trials on which the member of Group A, B, or C higher in perspective was selected were $.88, .98$, and .93 in Exp. I and .92, .97, and .95 in Exp. II. The proportions of pairs in Group D for which the member with higher slant indicated by the form ratio was selected were .59 and $.62 \mathrm{in}$ Exp. I and II, respectively.

An analysis of variance of the proportion of trials on which the member of a pair with the greater perspective was selected was

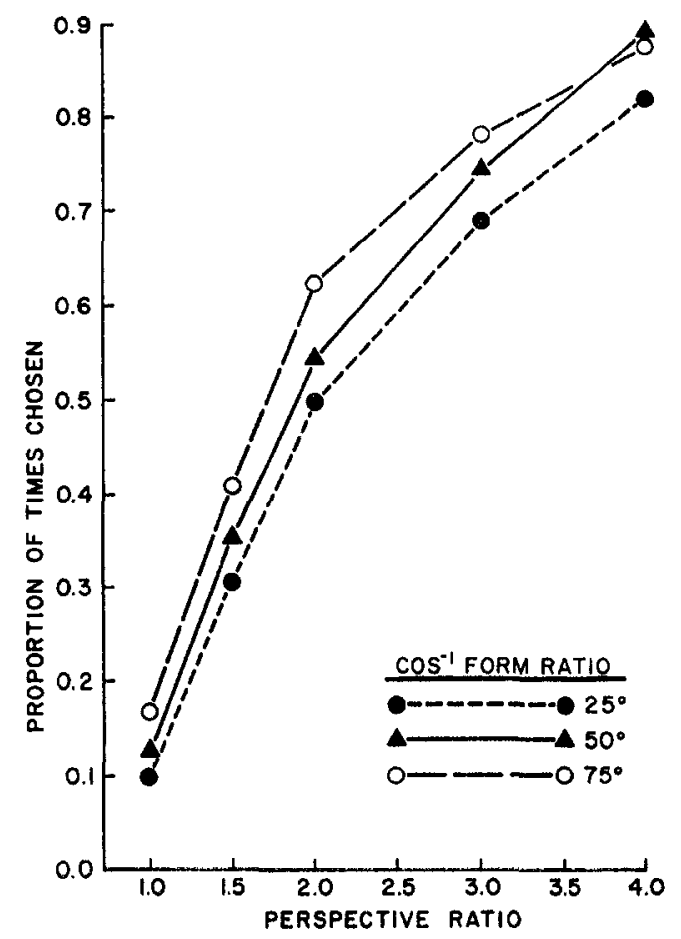

FIG. 3. Effect of perspective and form ratio on relative slant judgments for regular dot patterns.

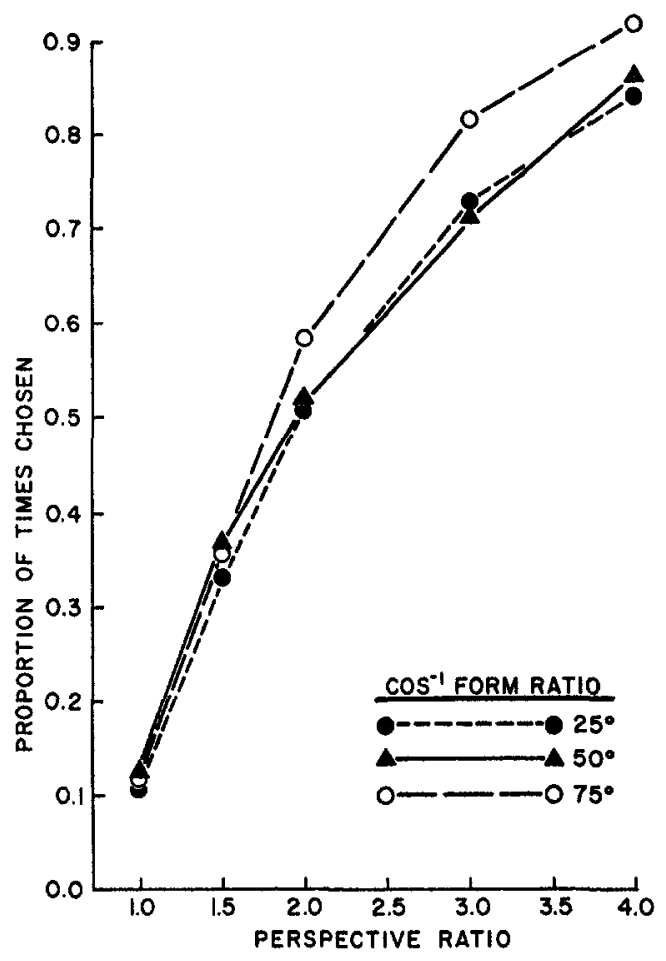

FIG. 4. Effect of perspective and form ratio on relative slant judgments for regular line patterns.

conducted for Groups A, B, and C, with group as a within- $S$ s variable and display type (regular dot patterns vs. line patterns) as a between- $S$ s variable. The main effect of groups was significant, $F(2,92)=10.8$, $p<.05$; the main effect of display types, $F(1,46)=1.4$, and its interaction with groups, $F(2,92)=.9$, were not. A comparison of the individual group means showed only $A$ and $B$ to be significantly different, with $C$ assuming an intermediate value.

A similar analysis was conducted for Groups A, B, and D. The dependent variable was the proportion of trials on which $S$ selected the display for which the form ratio indicated greater slant. The main effect of groups was again significant, $F(2,92)=$ $641.7, p<.05$, and the main effect of display types, $F(1,46)=.2$, and the interaction, $F(2,92)=.7$, were not. All pairs of group means differed significantly.

Experiment III.-The mean proportions of trials on which each display was selected 
as having the greater slant were $.30, .34, .46$, .64 , and .77 for perspective levels of $1.0,1.5$, $2.0,3.0$, and 4.0 , respectively. The mean proportion of trials on which $S$ s selected the stimulus displaying the greater perspective as having the greater slant was .72. This is significantly greater than chance $(.5), t(23)$ $=7.07, p<.05$, but less than the values obtained for pairs differing only in perspective in Exp. I (.93), $t(46)=5.7, p<.05$, and in Exp. II $(.95), t(46)=6.6, p<.05$. The mean judgment of greatest displayed slant $(N=24)$ was $20^{\circ}$.

\section{Discussion}

When perspective and form ratio are independently varied in regular dot and line patterns, perspective appears to be the principal variable underlying relative slant judgments. This was most clearly demonstrated when $S$ s were presented with pairs of displays in which one had a greater slant indicated by perspective and the other had a greater slant indicated by the form ratio. The display for which greater slant was indicated by perspective was generally chosen as appearing more slanted. If $S \mathrm{~s}$ had judged slant in accordance with an assumed original form in which the spacing between horizontal elements was equal to the spacing between vertical elements (as was the case for the demonstration plane visible to $S$ at the beginning of the experiment), the denominator of the form ratio would have been 1. Another denominator would have been appropriate if some other original form were assumed during the responses to the paired stimuli, but slant judgments should still have been ordered by the numerator of the form ratio. When the form ratio and perspective provided conflicting indications of relative slant, judgments were not ordered by the numerator of the form ratio, which is directly measurable in the stimuli. It must be concluded either that $S$ gave relatively little weight to the original form in making his judgments or that his assumption concerning the original form varied with perspective in the displayed projection. Either conclusion confirms the dominant role of perspective in these judgments. The form ratio did have some effect, however. The tendency to choose the display with greater perspective was increased when the form ratio reinforced rather than conflicted with the slant indications provided by perspective. When perspective was constant for both displays in a pair, the display for which the form ratio indicated greater slant was chosen with higher than chance probability.

The implications of slant judgments based on perspective are well known (Ittelson, 1960, Ch. 5). In artificial projections, e.g., photographs, the slant indicated by perspective varies with viewing distance, and accurate perception of slant in such projections depends on the presence of additional sources of depth information. In the absence of other depth information, slant perception based on perspective may be misleading.

In an interchange of papers, Flock (1965) and Freeman (1965, 1966b) have debated the relative merits of optical theta and perspective as explanations of slant perception. Freeman argues that optical theta requires too complex an analytical operation to be a likely perceptual process. Actually, the three elements in optical theta are directly available to $O$, and there is no reason to assume that he must compute a cosine in order to judge slant on the basis of these elements. These three elements necessarily determine a slope at a given distance from the fixation point along the axis of rotation. Slope indications are present even in random textures, where slope is measurable as the rate of change in texture element density in the projection of the texture. In an outline figure rotated about a horizontal axis, optical theta is based on the projections of three separations between vertical contours that are parallel in the unslanted figure. The explanations of slant perception postulated by Flock and Freeman are geometrically equivalent as long as the distance of the sloping contour line from the fixation point is considered in the latter case.

While perspective information is available in random as well as in regular textures, the tendency to select the display in a pair with the highest perspective ratio was significantly reduced for random dot patterns. Estimates of maximum displayed slant were similarly reduced. These findings are in agreement with those of Gibson and Gibson (1957). It is likely that converging lines or rows of dots provide an especially effective presentation of perspective information in contourless displays. When an overall contour is present, $S$ may use the angle of convergence as a heuristic in the perception of surface slant. 


\section{REFERENCES}

Braunstein, M. L. Motion and texture as sources of slant information. Journal of Experimental Psychology, 1968, 78, 247-253.

Ericksson, E. S. The shape slant invariance hypothesis in static perception. Scandinavian Journal of Psychology, 1967, 8, 193-208.

FLock, H. R. The monocular perception of surface slant. (Doctoral dissertation, Cornell University) Ann Arbor, Mich.: University Microfilms, 1962. No. 62-2514.

FLock, H. R. Optical texture and linear perspective as stimuli for slant perception. Psychological Review, 1965, 72, 505-514.

Freeman, R. B., JR. Ecological optics and visual slant. Psychological Review, 1965, 72, 501-504.
Freeman, R. B., JR. Function of cues in the perceptual learning of visual slant: An experimental and theoretical analysis. Psychological Monographs, 1966, 80 (2, Whole No. 810). (a)

Freeman, R. B., JR. Optical texture versus retinal perspective: A reply to Flock. Psychological Review, 1966, 73, 365-371. (b)

Gibson, J. J., \& Gibson, E. J. Continuous perspective transformations and the perception of rigid motion. Journal of Experimental Psychology, 1957, 54, 129-138.

ITTELSon, W. H. Visual space perception. New York: Springer, 1960.

(Received January 10, 1969)

\section{(Continued from page 583)}

Transfer of Eyelid Conditioning from Instrumental to Classical Reinforcement and Vice Versa: David A. Grant,* Neal E. A. Kroll, Barry Kantowitz, Michael J. Zajano, and Kenneth B. Solberg : Department of Psychology, Psychology Buidling, Charter at Johnson, University of Wisconsin, Madison, Wisconsin 53706.

Effect of Varying Channel Capacity on Stimulus Detection and Discrimination: Marilyn C. Smith*: Department of Psychology, University of Toronto, Toronto 5, Canada.

Role of Semantics in Remembering Comparative Sentences: Herbert H. Clark* and Stuart K. Card: Department of Psychology, Schenley Park, Carnegie-Mellon University, Pittsburgh, Pennsylvania 15213.

Number of Food Pellets and the Partial Reinforcement Extinction Effect after Extended Acquisition: Abram Amsel,* C. Thomas Surridge, and James J. Hug: Department of Psychology, University of Toronto, Toronto 5, Canada.

Response Latency as a Function of Interstimulus Interval in Conditioned Eyelid Discrimination: William E. Vandament*: Department of Psychology, State University of New York at Binghamton, Binghamton, New York 13901.

Choice Response Times as Functions of Intralist Similarity, Stimulus Type, and Number of Equally Probable Alternatives: Barry Gholson* and Raymond H. Hohle: Department of Psychology, State University of New York at Stony Brook, Stony Brook, New York 11790.

Joint Effects of Proactive and Retroactive Interference as a function of Degree of Learning: Theresa S. Howe*: Department of Psychology, University of Missouri, 3001 Natural Bridge Road, Saint Louis, Missouri 63121.

Conditions of Recovery after Unlearning: Leo Postman,* Karen Stark, and Diane Henschel: Institute of Human Learning, University of California, Berkeley, California 94720.

Stimulus Control and Memory Loss in the Reversal-Shift Behavior of College Students: Howard H. Kendler," Tracy S. Kendler, and Richard S. Marken: Center for Advanced Study in the Behavioral Sciences, 202 Junipero Serra Boulevard, Stanford, California 94305.

* Asterisk indicates author for whom address is given. 